\title{
Zonula Occludens-1 and -2 Are Cytosolic Scaffolds That Regulate the Assembly of Cellular Junctions
}

\author{
Alan S. Fanning and James M. Anderson \\ The Department of Cell and Molecular Physiology, University of North Carolina at Chapel Hill, \\ Chapel Hill, North Carolina, USA
}

\begin{abstract}
The integrity of the tight junction barrier in epithelial and endothelial cells is critical to human health, but we still lack a detailed mechanistic knowledge of how the barrier is formed during development or responds to pathological and pharmacological insults. This limits our understanding of barrier dysfunction in disease and slows the development of therapeutic strategies. Recent studies confirm the long-maintained but previously unsupported view that the zonula occludens (ZO) proteins ZO-1 and ZO-2 are critical determinants of barrier formation. However, ZO proteins can also be components of adherens junctions, and recent studies suggest that $\mathrm{ZO}$ proteins may also promote the assembly and function of these junctions during epithelial morphogenesis. We review these studies and outline several recent observations that suggest that one role of $\mathrm{ZO}$ proteins is to regulate cytoskeletal dynamics at cell junctions. Finally, we propose a model by which the functional activities of $\mathrm{ZO}$ proteins in the adherens junction and tight junction are differentiated by a novel regulatory motif known as the U6 or acidic motif.
\end{abstract}

\section{Keywords}

tight junction; adherens junction; zonula occludens; ZO-1; ZO-2; E-cadherin; cytoskeleton; MAGUK; PDZ; scaffold; permeability; epithelia; morphogenesis

\section{Assembly of Cell-Cell Junctions Is a Multistep Process}

Polarized epithelial cells are circumscribed at their apical-lateral margin by two morphologically distinct intercellular junctions that together form what is known as the apical junctional complex (AJC). The adherens junction (AJ) mediates cell-cell adhesion and signaling pathways that control growth, cell morphology, and differentiation. ${ }^{1}$ The tight junction (TJ) regulates the movement of ions, macromolecules, and immune cells through the paracellular space and is critical for ion transport. ${ }^{2,3}$

The assembly of the TJ barrier during morphogenesis or tissue repair is a multistep process that involves the initial establishment of adhesive cell-cell contacts, the extension of these contacts laterally along the cell-cell contact zone, and the subsequent (perhaps concurrent) recruitment of transmembrane proteins, such as claudins, junction adhesion molecule (JAM), and occludin/tricellulin that form the paracellular barrier. ${ }^{4,5}$ The initial contacts are isolated punctate structures formed by the adhesive activities of the cadherin-catenin

\section{(C) 2009 New York Academy of Sciences}

Address for correspondence: Alan S. Fanning, Department of Cell and Molecular Physiology, University of North Carolina at Chapel Hill CB7545, Chapel Hill, NC 27599-7545. Voice: +919-966-6412; fax: +919-966-6413. alan_fanning@med.unc.edu.

Conflicts of Interest

The authors declare no conflicts of interest. 
complex and can occur within filopodial structures or at discrete sites within a larger contact zone between two cellular membranes (reviewed in Ref. 6). These adhesive contacts are subsequently extended into a circumferential structure known as the zonula adherens (ZA) that circumscribes the apical-lateral boundary of polarized epithelial cells. This latter process is poorly understood, but current evidence supports the hypothesis it involves the coordinated actions of multiple signaling pathways ${ }^{7,8}$ with the cortical actin cytoskeleton.

\section{Zonula Occludens Proteins Regulate Tight Junction Assembly}

Recent observations from our group and others strongly suggest that the proteins zonula occludens (ZO)-1 and ZO-2, which are members of the membrane-associated guanylate kinase (MAGUK) homologue family of proteins, are critical regulators of TJ assembly. The TJ barrier is composed of transmembrane proteins, such as the claudins, occludin, and tricellulin, that are organized into continuous adhesive strands that circumscribe the apicallateral margin of polarized epithelia. ${ }^{9}$ Significantly, many of these transmembrane proteins are also distributed throughout the entire lateral plasma membrane but they are only organized into barrier strands at the apical junctional complex-suggesting there are spatially and temporally regulated steps in the assembly of these proteins into strands.

Recently, the Tsukita group demonstrated that depletion of both ZO-1 and ZO-2 in the EpH4 mammary epithelial cell line completely abrogated $\mathrm{TJ}$ assembly. ${ }^{10}$ Using a different approach, we have demonstrated that expression of ZO proteins that contain mutations in a highly conserved region, the U6 domain, in MDCK cells can trigger the formation of ectopically positioned TJ transmembrane protein strands on the lateral plasma membrane. ${ }^{11}$ These observations, taken together, indicate that the $\mathrm{ZO}$ proteins are required for the assembly of TJ transmembrane proteins, such as claudin, into barrier strands and direct the incorporation of these strands into the AJC.

More recently it has been demonstrated that targeted disruption of either ZO-1 or ZO-2 in mice results in embryonic lethality that is correlated with disruption of the paracellular barrier and the structure of cell junctions. ${ }^{12,13}$ These observations suggest that the ZO proteins have nonredundant functions during development, unlike their role in strand assembly. However, the mechanistic basis for the early embryonic lethality in these mice is still under investigation.

These observations raise several critical questions. First, how do ZO proteins bring about the coordinated assembly of barrier proteins, such as the claudins, into a highly organized circumferential barrier? Second, at what step in epithelial barrier morphogenesis do ZO proteins act? Third, how is the assembly of the barrier linked to the assembly of other junctional complexes, such as the AJ? Based on the results reviewed below, we speculate that ZO proteins act at several stages of assembly and that their role is based on three very important properties of the ZO MAGUKs: (1) their ability to act as a temporally and spatially regulated scaffold for other TJ proteins, such as occludin/tricellulin and claudin; (2) their ability to bind to and regulate components of the cortical actin cytoskeleton; and (3) their ability to promote cadherin-mediated cell-cell adhesion.

\section{ZO Proteins Are Multidomain Scaffolding Molecules}

How do the ZO proteins act as scaffolds? The three $\mathrm{ZO}$ proteins, ZO-1, -2 , and -3 , are multidomain polypeptides (Fig. 1). They have an $\mathrm{N}$-terminal half with a domain structure similar to other MAGUK proteins that contains 3 PSD-95/discs-large/Zonula occludens-1 (PDZ) domains, an Src homologty-3 (SH3) domain, and a region of homology to guanylate kinase (for review, see Refs. 9,14). Interspersed between these conserved protein-binding domains are so-called unique $(\mathrm{U})$ motifs. The protein-binding motifs within the $\mathrm{N}$ terminus 
mediate direct interactions with all of the knownTJ transmembrane proteins and many of the cytosolic proteins that localize to TJ. For example, the first PDZ domain (PDZ1) binds to claudins, PDZ3 binds to JAM proteins, and the U5+GUK domain binds to occludin and tricellulin. ${ }^{15-18}$ In ZO-1, like other MAGUKs, the U5 domain (also known as the HOOK domain), located between the SH3 and GUK domains, is required for junction localization, whereas the U6 domain regulates the activity of the U5 and GUK domains in a fashion not yet fully understood (discussed below). ${ }^{11}$ Interestingly, the AJ proteins a catenin, AF-6/ afadin, and ARVCF have also been reported to bind within the N-terminal half of ZO-1 and $\mathrm{ZO}-2 .{ }^{19-22}$ The possible functional relevance of this is considered below.

The C-terminal regions are unique to the three ZO MAGUKs and not present in proteins such as PSD95, CASK, and Dlg. Although highly divergent at the amino acid level, the Cterminal regions all bind directly to F-actin or with other actin-binding proteins, such as protein 4.1 and cortactin. ${ }^{17,23,24}$ Both AF-6/afadin and cingulin also appear to have binding sites in the $\mathrm{C}$ terminus. ${ }^{11,25}$

Importantly, the second PDZ domains of ZO MAGUKs mediate homodimerization and heterodimerization between these three molecules. ${ }^{26,27}$ Although the functional role of PDZ dimerization is poorly understood, one recent study suggested that cross-linking of $\mathrm{ZO}$ proteins within the SH3/GUK regions is critical for strand assembly. ${ }^{10}$ This is not without precedent; in the synaptic MAGUKS PSD-95 and SAP102, "domain swapping" between SH3 and GUK domains of these two proteins results in the formation of heterodimers. ${ }^{28}$ Thus, we predict that the ability of ZO proteins to promote the assembly of TJ proteins into the paracellular barrier not only depends upon their ability to bind directly to many different junctional proteins but also on an intrinsic ability to cross-link these proteins into larger arrays. The critical questions that arise include: What protein-protein interactions are necessary for assembly of $\mathrm{TJ}$ transmembrane proteins into strands and the incorporation of these strands into the AJC? Does multimerization play a role in the assembly of barrier strands? How is the temporal and spatial assembly of TJ proteins into strands controlled?

Very little is known about how binding of proteins to ZO-1 is regulated or how cell signaling pathways control TJ assembly and barrier function, although many different pathways have been implicated (for review, see Ref. 29). Interestingly, it was recently demonstrated that Ca-calmodulin binds to the GUK domain of ZO- $1,{ }^{30}$ suggesting the novel hypothesis that intracellular calcium levels control some ZO-1 functions. Again, such a mechanism is not without precedent. Ca-calmodulin binds within the U5 region of other MAGUKs, and this interaction regulates multimerization in vitro. ${ }^{28,30}$ Another regulator of $\mathrm{TJ}$ assembly, $\mathrm{Ga} 12$, binds to the $\mathrm{SH} 3$ domain and appears to regulate the interactions of ZO-1 with several TJ ligands. ${ }^{31}$ Thus, there are several lines of evidence that suggest that regulated interactions with the region encoding the $\mathrm{SH} 3$, U5, and GUK domains are critical for TJ assembly.

\section{Do ZO Proteins Regulate Adherens Junction Assembly and/or Cell-Cell Adhesion?}

It has been appreciated for several years that the assembly and integrity of TJs are intertwined with that of AJs. Disruption of E-cadherin-mediated cell-cell adhesion with extracellular antibodies to E-cadherin ${ }^{32}$ or small interfering (si)RNA ${ }^{33}$ delays TJ assembly. Furthermore, ZO-1 and ZO-2 localize to AJ with cadherin and catenins at the earliest punctuate cell-cell contacts prior to proteins such as occludin or claudin and bind directly to AJ proteins, such as a catenin, AVRCF, and AF-6/afadin. ${ }^{20,22,34,35}$ Thus, the prevalent hypothesis is that $\mathrm{ZO}$ proteins use direct interactions with $\mathrm{AJ}$ proteins as a positional signal 
early during AJ assembly and subsequently recruit $\mathrm{TJ}$ proteins into an apical complex that is ultimately segregated from the AJ.

While this appears to be one role for the $\mathrm{ZO}$ proteins, the evidence suggests they are actually active components in $\mathrm{AJ}$ formation or cell adhesion. $\mathrm{ZO}$ proteins are components of the AJ in nonepithelial cells, such as fibroblasts and cardiomyocytes, and are primarily localized to AJs in nonvertebrates, such as Drosophila and Caenorhabditis elegans. ${ }^{25,36-38}$ In addition, several groups have identified defects in AJ assembly in cells lacking $\mathrm{ZO}$ proteins. For example, the extension of AJ plaques into a circumferential belt was delayed by more than $24 \mathrm{~h}$ in ZO-deficient EpH4 cells, and disruption of the ZA was observed in fully polarized cells. ${ }^{39,40}$ Similarly, Hernandez et al. found that knockdown of ZO-2 alone was sufficient to briefly delay AJ assembly and disrupt E-cadherin localization in MDCK cells. ${ }^{41}$ Finally, the combination of weak mutations in the single Drosophila ZO protein PYD and the AF-6/ afadin homologue Canoe, which on their own have no AJ defect, have severe AJ defects when combined. ${ }^{25}$ Stronger Pyd alleles appear to have more dramatic alterations in adhesion that lead to defects in dorsal closure and eye morphogenesis. ${ }^{42,43}$ Significantly, exogenous PYD transgenes can only rescue AJ defects when they localize to the AJ, and this requires the U6 motif discussed below. ${ }^{42}$ These observations suggest that $\mathrm{ZO}$ proteins not only serve to recruit TJ proteins to the circumferential belt but they also directly regulate the activity of AJs.

How ZO proteins might regulate AJ assembly is a matter of speculation. ZO proteins do not appear to be required for the de novo assembly of cadherin or catenins into primordial AJ in cultured epithelial cells ${ }^{39,40}$ or for their localization to AJ in Drosophila embryos. ${ }^{25,43,44}$ Instead, they appear to regulate the extension of AJ proteins into a circumferential complex ${ }^{39}$ and morphogenetic processes, such as tubulogenesis ${ }^{44}$ and dorsal closure ${ }^{42}$ and tissue morphogenesis in the pupal eye. ${ }^{43}$ One hypothesis that might explain these observations is that $\mathrm{ZO}$ proteins can directly promote adhesion of the cadherin-catenin complex during dynamic reorganization of cell-cell junctions. This hypothesis is at least partly supported by the observation that E-cadherin/a catenin chimeras lacking the ZO-1 binding domain in a catenin cannot promote cell-cell adhesion in L cell fibroblasts lacking endogenous E-cadherin, although E-cadherin/a catenin chimeras can do so. ${ }^{45}$

\section{The U6 Motif Regulates the Assembly of Tight Junction Strands}

In epithelial cells, ZO proteins are necessary for the assembly of TJ proteins, such as claudin, into TJ strands. We hypothesize that the spatial, and perhaps temporal, positioning of these strands is dependent upon the U6 motif. This conserved motif, which is unique to ZO MAGUKs, is immediately $\mathrm{C}$ terminal to the GUK domains in all ZO proteins (Fig. 1). We have demonstrated that this motif negatively regulates incorporation of ZO-1 into the $\mathrm{TJ}$, that it inhibits the binding of occludin to $\mathrm{ZO}-1$, and that deletion of this motif results in assembly of ectopically positioned strands. ${ }^{11}$ These results were confirmed by Ikenouchi et al. ${ }^{39}$ who demonstrated that this motif is required for the localization of TJ strands to the AJC in ZO-1/ZO-2-depleted cells. ZO-1 transgenes that lack the U6 domain can initiate the polymerization of claudins on the lateral surface of $\mathrm{ZO} 1 / \mathrm{ZO} 2$-depleted cells but they are unable to incorporate claudins into a circumferential barrier. ${ }^{39}$ Thus, the U6 motif regulates the positioning of TJ strands in the AJC as well as the incorporation of transmembrane proteins, such as occludin or tricellulin, into these strands.

Our published observations indicate that the U6 motif does not directly target ZO-1 to the AJC. Instead, sequences within the U5 motif appear to be necessary and sufficient for TJ localization, much like the U5 domain (also known as the "HOOK") in other MAGUK proteins. ${ }^{11,46,47}$ Thus, the ability of the U6 motif to inhibit TJ localization suggests that the 
U6 domain somehow regulates the activity of the U5 motif. In a similar manner, the inhibition of occludin binding suggests that U6 also inhibits the binding of some proteins to the GUK domain. Interestingly, the U6 domain is also necessary for ZA assembly in ZO-1/ ZO-2-depleted cells; ZO-1 transgenes lacking the U6 motif cannot rescue the defect in AJ assembly in these cells. ${ }^{39,40}$ Thus, the U6 domain appears to act as a bifunctional switch that inhibits TJ assembly (incorporation into AJC and binding to occludin/tricellulin) but promotes AJ assembly. We propose that the U6 motif distinguishes between the dual activities of $\mathrm{ZO}$ proteins in the $\mathrm{AJ}$ and $\mathrm{TJ}$ (discussed below) and suggest that it regulates the segregation of $\mathrm{AJ}$ and $\mathrm{TJ}$ proteins that develops within the AJC of polarized epithelia.

\section{ZO-1 Regulates the Cortical Cytoskeleton at Cell-Cell Contacts}

A third hypothesis currently being tested in our laboratory is that ZO-1 regulates both AJ and $\mathrm{TJ}$ function by coordinating the assembly or dynamics of the cortical cytoskeleton. Both TJs and AJs are intimately associated with the cortical actin cytoskeleton, and there is a considerable body of evidence that indicates that the assembly and functional activity of cell-cell junctions relies on the integrity of the cytoskeletal arrays with which they are physically associated. ${ }^{7,9} \mathrm{AJ}$ proteins like a catenin, AF-6, and vinculin all bind directly to Factin and regulate actin dynamics in vitro, and disruption of cytoskeletal interactions with these proteins can clearly disrupt AJ assembly and cell-cell adhesion. ${ }^{21,45,48,49}$ The assembly of the TJ, like the AJ, is also sensitive to physiological and pharmacological regulators of cytoskeletal dynamics, and ZO proteins bind directly to F-actin and to several proteins that can regulate cytoskeletal dynamics (reviewed in Ref. 50). For example, both ZO-1 and ZO-2 bind to cortactin, which promotes and stabilizes the assembly of branched filaments at the plasma membrane. ${ }^{24} \mathrm{ZO}-1$ has also been demonstrated to interact with vasodilatorstimulated phosphoprotein (VASP), ${ }^{51}$ protein $4.1,{ }^{23}$ shroom $2,{ }^{52}$ and the Cdc42 guanine nucleotide exchange factor (GEF) Tuba. ${ }^{53}$

Disruption of ZO protein expression has a dramatic effect on cytoskeletal dynamics at AJ and TJ. In MDCK cells, depletion of ZO-1 alters the dynamics of F-actin redistribution following calcium switch, ${ }^{54}$ and depletion of ZO-2 alone disrupts F-actin structures associated with junctions. ${ }^{41}$ More dramatically, depletion of both ZO-1 and ZO-2 in EpH4 cells delays the formation of circumferential actin bundles by $72 \mathrm{~h}$, eliminates the band of Factin that is normally tightly associated with the $\mathrm{ZA}$, and disrupts the incorporation of myosin II into AJ ${ }^{39,40}$ Finally, loss of the single ZO-1 orthologue in C. elegans disrupts the accumulation of F-actin at AJ during embryogenesis. ${ }^{38}$ These observations support the hypothesis that $\mathrm{ZO}$ proteins, or their binding partners, are regulators of F-actin dynamics at cellular junctions.

The functional/mechanistic relevance of these cytoskeletal interactions with $\mathrm{ZO}$ proteins to $\mathrm{TJ}$ or AJ biology is still poorly understood. One possibility is that ZO proteins passively link transmembrane proteins, such as claudin, occluding, or the cadherin/catenin complex, to the cortical cytoskeletal and that this has some role in stabilizing junctional complexes in the plasma membrane. An alternative possibility is that $\mathrm{ZO}$ proteins directly regulate the assembly or dynamics of F-actin at $\mathrm{AJ}$ or TJ and may thus regulate the downstream morphogenetic processes of epithelia, such as tubulogenesis, wound repair, or epithelialmesenchymal transitions. A recent study demonstrated that ZO-1 was required for threedimensional cyst formation in MDCK cells, ${ }^{55}$ and Wittchen et al. found an increased rate of wound closure in cells expressing ZO-3 truncation mutants. ${ }^{56}$ It has also been demonstrated that ZO-1 is required for localization of Tuba and myosin II to cell junctions. ${ }^{40,53}$ Disruption of Tuba expression delays junction assembly, alters junctional F-actin, and results in changes in cell shape. ${ }^{53}$ Finally, Ikenouchi et al. found that rac activation, which is critical for cytoskeletal dynamics, was markedly reduced in $\mathrm{ZO} 1 / \mathrm{ZO} 2$ knockdown cells. ${ }^{39}$ Together 
these results support the hypothesis that ZO proteins recruit and/or activate the machinery of actin filament assembly at junctions during critical points in epithelial morphogenesis.

\section{Conclusion}

Recent studies have confirmed the long-held view that $\mathrm{ZO}$ proteins are critical regulators of $\mathrm{TJ}$ assembly and have presented the novel hypothesis that $\mathrm{ZO}$ proteins may also promote $\mathrm{AJ}$ assembly. The critical elements of these functions are likely to be the ability of ZO proteins to bind to and cross-link other junction proteins into higher order arrays and to link these arrays to the underlying cortical cytoskeleton. Whether $\mathrm{ZO}$ proteins have a more active role in the dynamics of this cytoskeletal array remains to be determined. It is also not clear if or how the various signaling pathways that regulate junction assembly might work through $\mathrm{ZO}$ proteins. However, it is clear that the U6 motif is an important regulatory element in $\mathrm{ZO}$ proteins that warrants further study in the context of these signaling pathways.

\section{References}

1. Halbleib JM, Nelson WJ. Cadherins in development: cell adhesion, sorting, and tissue morphogenesis. Genes Dev. 2006; 20:3199-3214. [PubMed: 17158740]

2. Anderson JM, Van Itallie CM, Fanning AS. Setting up a selective barrier at the apical junction complex. Curr. Opin. Cell Biol. 2004; 16:140-145. [PubMed: 15196556]

3. Lee DBN, Huang E, Ward HJ. Tight junction biology and kidney dysfunction. Am. J. Physiol. Renal Physiol. 2006; 290:F20-F34. [PubMed: 16339962]

4. Adams CL, Nelson WJ. Cytomechanics of cadherin-mediated cell-cell adhesion. Curr. Opin. Cell Biol. 1998; 10:572-577. [PubMed: 9818166]

5. Ebnet K, Suzuki A, Ohno S, Vestweber D. Junctional adhesion molecules (JAMs): more molecules with dual functions? J. Cell Sci. 2004; 117:19-29. [PubMed: 14657270]

6. Vasioukhin V, Fuchs E. Actin dynamics and cell-cell adhesion in epithelia. Curr. Opin. Cell Biol. 2001; 13:76-84. [PubMed: 11163137]

7. Yap AS, Ali R, Goodwin M, et al. E-cadherin signaling to the actin assembly machinery is necessary for dynamic adhesive recognition. Mol. Cell Biol. 2001; 12:349A-350A.

8. Miyoshi J, Takai Y. Molecular perspective on tight-junction assembly and epithelial polarity. Adv. Drug Deliv. Rev. 2005; 57:815-855. [PubMed: 15820555]

9. Fanning, AS. ZO Proteins and tight junction assembly. In: Gonzalez-Mariscal, L., editor. Tight Junctions. New York: Landes Bioscience; 2006. p. 64-65.

10. Umeda K, Ikenouchi J, Katahira-Tayama S, et al. ZO-1 and ZO-2 independently determine where claudins are polymerized in tight-junction strand formation. Cell. 2006; 126:741-754. [PubMed: 16923393]

11. Fanning AS, Little BP, Rahner C, et al. The unique-5 and-6 motifs of ZO-1 regulate tight junction strand localization and scaffolding properties. Mol. Cell Biol. 2007; 18:721-731.

12. Xu J, Kausalya PJ, Phua DC, et al. Early embryonic lethality of mice lacking ZO-2, but not ZO-3, reveals critical and non-redundant roles for individual $\mathrm{ZO}$ proteins in mammalian development. Mol. Cell Biol. 2008; 28:1669-1678. [PubMed: 18172007]

13. Katsuno T, Umeda K, Matsui T, et al. Deficiency of zonula occludens-1 causes embryonic lethal phenotype associated with defected yolk sac angiogenesis and apoptosis of embryonic cells. Mol. Cell Biol. 2008; 19:2465-2475.

14. Schneeberger EE, Lynch RD. The tight junction: a multifunctional complex. Am. J. Physiol. Cell Physiol. 2004; 286:C1213-C1228. [PubMed: 15151915]

15. Itoh M, Furuse M, Morita K, et al. Direct binding of three tight junction-associated MAGUKs, ZO-1, ZO-2, and ZO-3, with the COOH termini of claudins. J. Cell Biol. 1999; 147:1351-1363. [PubMed: 10601346] 
16. Ebnet K, Schulz CU, Meyer Zu, Brickwedde MK, et al. Junctional adhesion molecule interacts with the PDZ domain-containing proteins AF-6 and ZO-1. J. Biol. Chem. 2000; 275:2797927988. [PubMed: 10856295]

17. Fanning AS, Ma TY, Anderson JM. Isolation and functional characterization of the actinbinding region in the tight junction protein ZO-1. Faseb Journal. 2002; 16:1835-1837. [PubMed: 12354695]

18. Muller SL, Portwich M, Schmidt A, et al. The tight junction protein occludin and the adherens junction protein alpha-catenin share a common interaction mechanism with ZO-1. J. Biol. Chem. 2005; 280:3747-3756. [PubMed: 15548514]

19. Itoh M, Nagafuchi A, Moroi S, Tsukita S. Involvement of ZO-1 in cadherin-based cell adhesion through its direct binding to alpha catenin and actin filaments. J. Cell Biol. 1997; 138:181-192. [PubMed: 9214391]

20. Yamamoto T, Harada N, Kano K, et al. The Ras target AF-6 interacts with ZO-1 and serves as a peripheral component of tight junctions in epithelial cells. J. Cell Biol. 1997; 139:785-795. [PubMed: 9348294]

21. Mandai K, Nakanishi H, Satoh A, et al. Afadin: A novel actin filament-binding protein with one PDZ domain localized at cadherin-based cellto-cell adherens junction. J. Cell Biol. 1997; 139:517-528. [PubMed: 9334353]

22. Kausalya PJ, Phua DC, Hunziker W. Association of ARVCF with ZO-1 and ZO-2: binding to PDZ-domain proteins and cell-cell adhesion regulate plasma membrane and nuclear localization of ARVCF. Mol. Biol. Cell. 2004; 15:5503-5515. [PubMed: 15456900]

23. Mattagajasingh SN, Huang SC, Hartenstein JS, Benz EJJ. Characterization of the interaction between protein 4.1R and ZO-2. A possible link between the tight junction and the actin cytoskeleton. J. Biol. Chem. 2000; 275:30573-30585. [PubMed: 10874042]

24. Katsube T, Takahisa M, Ueda R, et al. Cortactin associates with the cell-cell junction protein ZO-1 in both Drosophila and mouse. J. Biol. Chem. 1998; 273:29672-29677. [PubMed: 9792678]

25. Takahashi K, Matsuo T, Katsube T, et al. Direct binding between two PDZ domain proteins Canoe and ZO-1 and their roles in regulation of the Jun N-terminal kinase pathway in Drosophila morphogenesis. Mech. Dev. 1998; 78:97-111. [PubMed: 9858699]

26. Utepbergenov DI, Fanning AS, Anderson JM. Dimerization of the scaffolding protein ZO-1 through the second PDZ domain. J. Biol. Chem. 2006; 281:24671-24677. [PubMed: 16790439]

27. Fanning AS, Jameson BJ, Jesaitis LA, Anderson JM. The tight junction protein ZO-1 establishes a link between the transmembrane protein occludin and the actin cytoskeleton. J. Biol. Chem. 1998; 273:29745-29753. [PubMed: 9792688]

28. Masuko N, Makino K, Kuwahara H, et al. Interaction of NE-dlg/SAP102, a neuronal and endocrine tissue-specific membrane-associated guanylate kinase protein, with calmodulin and PSD-95/SAP90. A possible regulatory role in molecular clustering at synaptic sites. J. Biol. Chem. 1999; 274:5782-5790. [PubMed: 10026200]

29. Matter K, Balda MS. Signalling to and from tight junctions. Nat. Rev. Mol. Cell Biol. 2003; 4:225-236. [PubMed: 12612641]

30. Paarmann I, Lye MF, Lavie A, Konrad M. Structural requirements for calmodulin binding to kinase ho-mologs. Protein Sci. 2008; 17:1946-1954. [PubMed: 18809851]

31. Sabath E, Negoro H, Beaudry S, et al. G\{alpha 12 regulates protein interactions within the MDCK cell tight junction and inhibits tight-junction assembly. J. Cell Sci. 2008; 1221:814-824. [PubMed: 18285450]

32. Gumbiner B, Stevenson B, Grimaldi A. The role of the cell adhesion molecule uvomorulin in the formation and maintenance of the epithelial junctional complex. J. Cell Biol. 1988; 107:15751587. [PubMed: 3049625]

33. Capaldo CT, Macara IG. Depletion of E-cadherin disrupts establishment but not maintenance of cell junctions in Madin-Darby canine kidney epithelial cells. Mol. Cell Biol. 2007; 18:189-200.

34. Stevenson BR, Siliciano JD, Mooseker MS, Goodenough DA. Identification of ZO-1: a high molecular weight polypeptide associated with the tight junction (zonula occludens) in a variety of epithelia. J. Cell Biol. 1986; 103:755-766. [PubMed: 3528172] 
35. Yonemura S, Itoh M, Nagafuchi A, Tsukita S. Cell-to-cell adherens junction formation and actin filament organization: similarities and differences between non-polarized fibroblasts and polarized epithelial cells. J. Cell Sci. 1995; 108:127-142. [PubMed: 7738090]

36. Jesaitis LA, Goodenough DA. Molecular characterization and tissue distribution of ZO-2, a tight junction protein homologous to ZO-1 and the Drosophila discs-large tumor suppressor protein. J. Cell Biol. 1994; 124:949-961. [PubMed: 8132716]

37. Howarth AG, Hughes MR, Stevenson BR. Detection of the tight junction-associated protein ZO-1 in astrocytes and other nonepithelial celltypes. Am. J. Physiol. 1992; 262:C461-C469. [PubMed: 1539634]

38. Lockwood C, Zaidel-Bar R, Hardin J. The C-elegans zonula occludens ortholog cooperates with the cadherin complex to recruit actin during morphogenesis. Curr. Biol. 2008; 18:1333-1337. [PubMed: 18718757]

39. Ikenouchi J, Umeda K, Tsukita S, et al. Requirement of ZO-1 for the formation of belt-like adherens junctions during epithelial cell polarization. J. Cell Biol. 2007; 176:779-786. [PubMed: 17353356]

40. Yamazaki Y, Umeda K, Wada M, et al. ZO-1- and ZO-2-dependent integration of myosin-2 to epithelial zonula adherens. Mol. Cell Biol. 2008; 19:3801-3811.

41. Hernandez S, Munguia BC, Gonzalez-Mariscal L. ZO-2 silencing in epithelial cells perturbs the gate and fence function of tight junctions and leads to an atypical monolayer architecture. Exp. Cell Res. 2007; 313:1533-1547. [PubMed: 17374535]

42. Wei XR, Ellis HM. Localization of the Drosophila MAGUK protein Polychaetoid is controlled by alternative splicing. Mech. Dev. 2001; 100:217-231. [PubMed: 11165479]

43. Seppa MJ, Johnson RI, Bao S, Cagan RL. Polychaetoid controls patterning by modulating adhesion in the Drosophila pupal retina. Dev. Biol. 2008; 318:1-16. [PubMed: 18423436]

44. Jung AC, Ribeiro C, Michaut L, et al. Polychaetoid/ZO-1 is required for cell specification and rearrangement during Drosophila tracheal morphogenesis. Curr. Biol. 2006; 16:1224-1231. [PubMed: 16782014]

45. Imamura Y, Itoh M, Maeno Y, et al. Functional domains of alpha-catenin required for the strong state of cadherin-based cell adhesion. J. Cell Biol. 1999; 144:1311-1322. [PubMed: 10087272]

46. Thomas U, Ebitsch S, Gorczyca M, et al. Synaptic targeting and localization of discs-large is a stepwise process controlled by different domains of the protein. Curr. Biol. 2000; 10:1108-1117. [PubMed: 10996791]

47. Hough CD, Woods DF, Park S, Bryant PJ. Organizing a functional junctional complex requires specific domains of the Drosophila MAGUK discs large. Genes Dev. 1997; 11:3242-3253. [PubMed: 9389655]

48. Zhadanov AB, Provance DWJ, Speer CA, et al. Absence of the tight junctional protein AF-6 disrupts epithelial cell-cell junctions and cell polarity during mouse development. Curr. Biol. 1999; 9:880-888. [PubMed: 10469590]

49. Watabe-Uchida M, Uchida N, Imamura Y, et al. alpha-catenin-vinculin interaction functions to organize the apical junctional complex in epithelial cells. J. Cell Biol. 1998; 142:847-857. [PubMed: 9700171]

50. Fanning, AS. Organization and regulation of the tight junction by the actin-myosin cytoskeleton. In: Anderson, JM.; Cereijido, M., editors. Tight Junctions. Boca Raton: CRC Press; 2001. p. 265-284.

51. Lawrence DW, Comerford KM, Colgan SP. Role of VASP in reestablishment of epithelial tight junction assembly after Ca2+ switch. Am. J. Physiol. Cell Physiol. 2002; 282:C1235-C1245. [PubMed: 11997237]

52. Etournay R, Zwaenepoel I, Perfettini I, et al. Shroom2, a myosin-VIIa-and actin-binding protein, directly interacts with ZO-1 at tight junctions. J. Cell Sci. 2007; 120:2838-2850. [PubMed: 17666436]

53. Otani T, Ichii T, Aono S, Takeichi M. Cdc42 GEF Tuba regulates the junctional configuration of simple epithelial cells. J. Cell Biol. 2006; 175:135-146. [PubMed: 17015620] 
54. McNeil E, Capaldo CT, Macara IG. Zonula occludens-1 function in the assembly of tight junctions in Madin-Darby canine kidney epithelial cells. Mol. Biol. Cell. 2006; 17:1922-1932. [PubMed: $16436508]$

55. Sourisseau T, Georgiadis A, Tsapara A, et al. Regulation of PCNA and cyclin D1 expression and epithelial morphogenesis by the ZO-1-regulated transcription factor ZONAB/DbpA. Mol. Cell Biol. 2006; 26:2387-2398. [PubMed: 16508013]

56. Wittchen ES, Haskins J, Stevenson BR. NZO-3 expression causes global changes to actin cytoskeleton in Madin-Darby canine kidney cells: Linking a tight junction protein to Rho GTPases. Mol. Cell Biol. 2003; 14:1757-1768. 
AF-6/afadin, cingulin, tricellulin

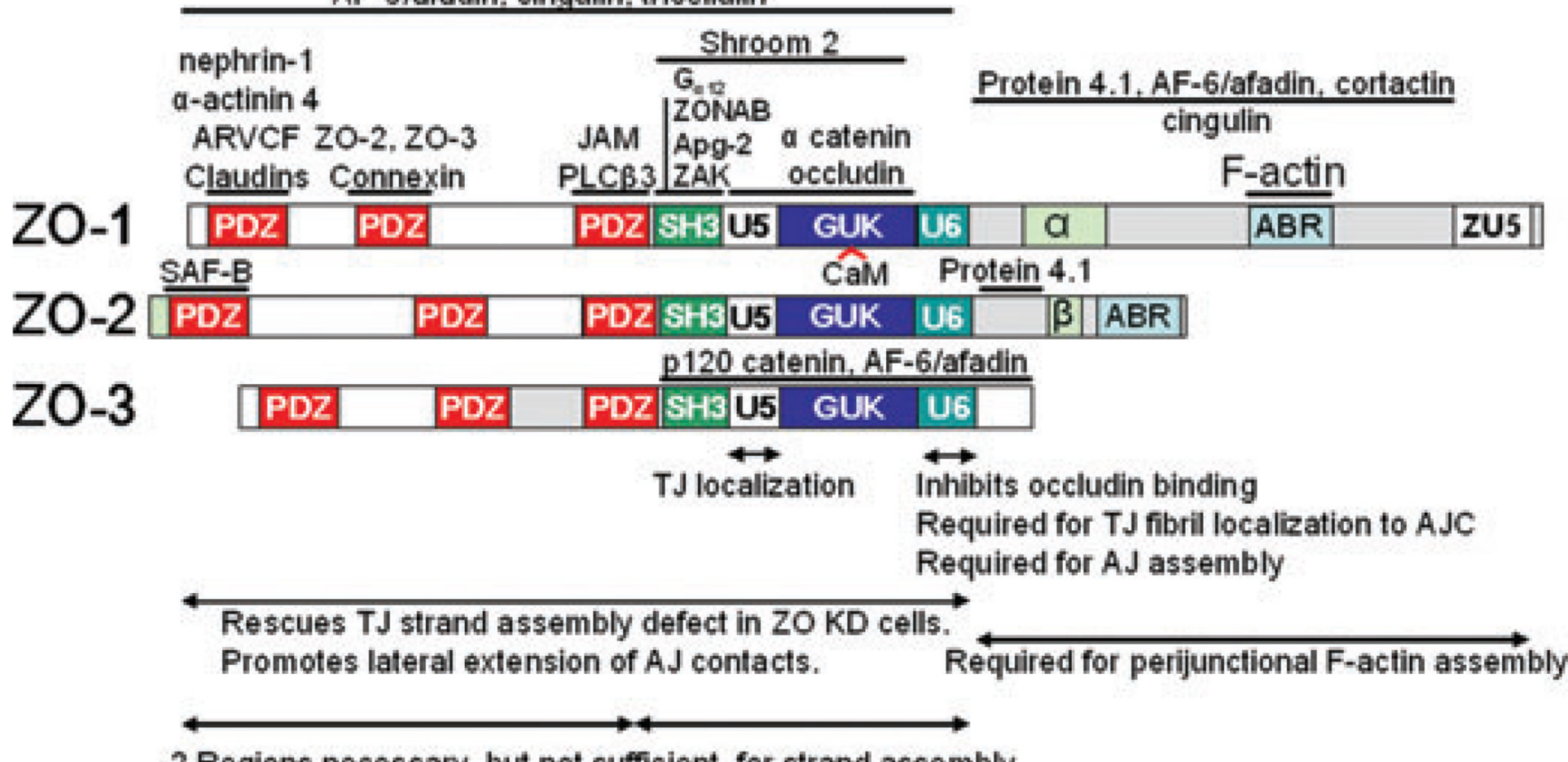

2 Regions necessary, but not sufficient, for strand assembly.

Figure 1.

Zonula occludens $(\mathrm{ZO})$ proteins are multidomain scaffolds required for tight junction (TJ) assembly. Protein ligands and their binding sites on ZO-1 (top), ZO-2, and ZO-3. The functional roles of different regions are indicated (bottom). See text for details. ABR, actinbinding region; ARVCF, armadillo repeat gene deleted in velo-cardiofacial syndrome; $\mathrm{CaM}$, calmodulin; GUK, guanylate kinase-like domain; PDZ, PSD-95/discs-large/zonula occludens-1 domain; PLCB3, phospholipase-C B3; SAF-B, scaffold attachment factor-B; SH3, Src Homology-3 domain; ZAK, zonula occludens kinase; ZONAB, ZO-1-associated nucleic acid binding protein; $\alpha, \beta$, and ZU5, alternatively spliced domains of unknown function. 